\title{
Discrete Frequency Warped Wavelets: Theory and Applications
}

\author{
Gianpaolo Evangelista, Member, IEEE, and Sergio Cavaliere
}

\begin{abstract}
In this paper, we extend the definition of dyadic wavelets to include frequency warped wavelets. The new wavelets are generated and the transform computed in discrete-time by alternating the Laguerre transform with perfect reconstruction filterbanks. This scheme provides the unique implementation of orthogonal or biorthogonal warped wavelets by means of rational transfer functions. We show that the discrete-time warped wavelets lead to well-defined continuous-time wavelet bases, satisfying a warped form of the two-scale equation. The shape of the wavelets is not invariant by translation. Rather, the "wavelet translates" are obtained from one another by allpass filtering. We show that the phase of the delay element is asymptotically a fractal. A feature of the warped wavelet transform is that the cut-off frequencies of the wavelets may be arbitrarily assigned while preserving a dyadic structure. The new transform provides an arbitrary tiling of the time-frequency plane, which can be designed by selecting as little as a single parameter. This feature is particularly desirable in cochlear and perceptual models of speech and music, where accurate bandwidth selection is an issue. As our examples show, by defining pitch-synchronous wavelets based on warped wavelets, the analysis of transients and denoising of inharmonic pseudo-periodic signals is greatly enhanced.
\end{abstract}

Index Terms-Quadrature mirror filters, time-frequency analysis, wavelet transforms.

\section{INTRODUCTION}

$\mathbf{I}$ $\mathrm{N}$ THIS paper, we consider extensions of the wavelet theory, defining a class of wavelets that provide a flexible orthogonal or biorthogonal tiling of the time-frequency plane. While in ordinary dyadic wavelets the frequency axis is partitioned in octave bands, in our class of transforms, this partition can be designed by selecting a set of parameters directly related to the cutoff frequencies of the wavelets. If the latter are constrained by a specific law, e.g., exponential, a single parameter is required. A considerable effort to enhance and adapt the frequency resolution of wavelets has been made in recent years by defining wavelets based on rational sampling rate, wavelet packets, and multiwavelets [23]-[26]. In these schemes, the "mother wavelet" is not unique. As a result, the design procedures become increasingly more complex as one increases the number of desired bands.

Manuscript received February 15, 1997; revised November 30, 1997. The associate editor coordinating the review of this paper and approving it for publication was Dr. Ahmed Tewfik.

The authors are with the Department of Physical Sciences, University Federico II, Napoli, Italy (e-mail: evangelista@na.infn.it).

Publisher Item Identifier S 1053-587X(98)02810-4.
The new bases are obtained by combining discrete Laguerre expansions with quadrature mirror filterbanks [6], [22]. As we will show in Section II, this is equivalent to frequency warping and filtering the wavelets. A class of continuoustime frequency warped wavelets were introduced in [12] and [13]. Our wavelets are the unique frequency warped wavelets that can be generated and the transform computed by means of rational transfer functions. By extending the construction of ordinary dyadic wavelets, we show that our discrete-time wavelets lead to well-defined continuous-time warped wavelets, provided that their defining infinite product converges pointwise. By means of warping, nonoctave bands are accommodated in the dyadic structure. Unlike ordinary wavelets, the new bases are not translation invariant at each fixed scale. However, the "translated wavelet" is obtained from the "mother wavelet" by allpass filtering. The usual unit delay elements are substituted by first-order allpass filters. We show that the phase of the iterated warping wavelets is a fractal.

Laguerre sequences and wavelets may be combined in several ways, each leading to a different complete and orthogonal or biorthogonal set. The simplest frequency warped wavelet expansions may be obtained by prewarping the signal by means of Laguerre expansion and by expanding the Laguerre coefficients onto a wavelet basis. In this case, a single parameter controls the tiling. These expansions may be extended by intertwining Laguerre expansion and QMF analysis. Using this scheme, one obtains the general case of wavelets with arbitrary bandwidths.

We also extend frequency warping to pitch-synchronous wavelets. Interesting applications arise in the analysis of pseudo-periodic signals whose partials are not uniformly spaced in the frequency domain. This is the case of quasistationary waves in dispersive media. The analysis of transients in strings, where dispersion is caused by stiffness - this phenomenon is apparent in piano strings in the low octave region-is greatly enhanced by using the pitch-synchronous warped wavelets. Furthermore, by building the pitch-synchronous bases on warped wavelets rather than on ordinary wavelets, one can increase at will the frequency resolution of the sidebands of the partials, enhancing feature extraction and signal separation.

The paper is organized in the following way. In Section II, we define the frequency warped filterbank, which is the building block of our warped wavelets. In Section III, we consider the iteration of the warped filterbank leading to discrete-time warped wavelets. The extension to continuous-time wavelets 


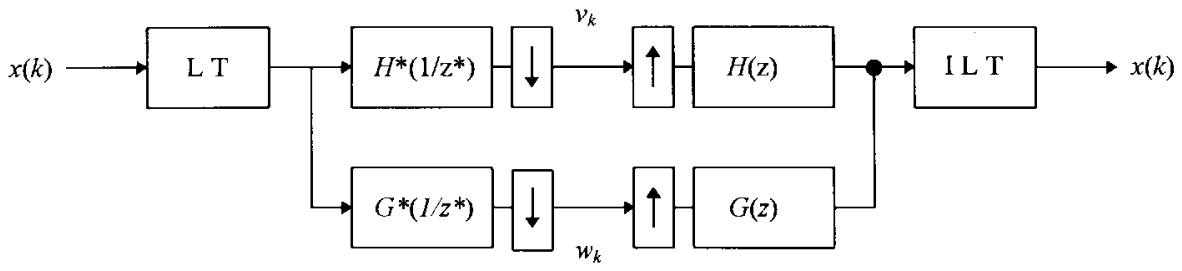

Fig. 1. Frequency warped filterbank structure including the Laguerre transform and inverse Laguerre transform blocks.

is explored in Section IV, where we consider two particular choices of the Laguerre parameters. In Section V, we look at some applications, presenting a specific example where warping enhances the analysis of inharmonic signals. Finally, in the Appendix, we present a brief review of the Laguerre transform and its implementation.

\section{FREQUENCY WARPED FILTERBANK}

The basic building block for computing the discrete frequency warped wavelet transform is given by the frequency warped filterbank, which is shown in Fig. 1. The analysis section is formed by cascading a Laguerre transform (LT) block, which is detailed in the Appendix, with a perfect reconstruction (PR) orthogonal filterbank [16], [17]. Accordingly, the synthesis section consists of the PR synthesis filterbank cascaded by the corresponding inverse Laguerre transform (ILT) block. The analysis structure performs the task of projecting the signal $x(k)$ onto the discrete Laguerre basis $\left\{\lambda_{r}\right\}_{r \in N}$ and then projecting the Laguerre expansion coefficients over the orthogonal sets associated with the filterbank. The latter sets are obtained by translating the two QMF impulse responses $h$ (lowpass) and $g$ (highpass) over the even integers: $h_{m}(k)=$ $h(k-2 m)$, and $g_{m}(k)=g(k-2 m) ; k, m$ integers. Denoting the Laguerre expansion coefficients by $x_{r}$ and the outputs of the filterbank by $v_{1, m}$ and $w_{1, m}$, respectively, for the lowpass and highpass branch, we obtain

$$
v_{1, m}=\sum_{r=0}^{\infty} h(r-2 m) x_{r}=\sum_{k=0}^{\infty} x(k) \varphi_{1, m}(k)
$$

and

$$
w_{1, m}=\sum_{r=0}^{\infty} g(r-2 m) x_{r}=\sum_{k=0}^{\infty} x(k) \psi_{1, m}(k)
$$

where

$$
x_{r}=\sum_{k=0}^{\infty} x(k) \lambda_{r}(k)
$$

and we defined

$$
\varphi_{1, m}(k) \equiv \sum_{r=0}^{\infty} \lambda_{r}(k) h_{m}(r)
$$

and

$$
\psi_{1, m}(k) \equiv \sum_{r=0}^{\infty} \lambda_{r}(k) g_{m}(r)
$$

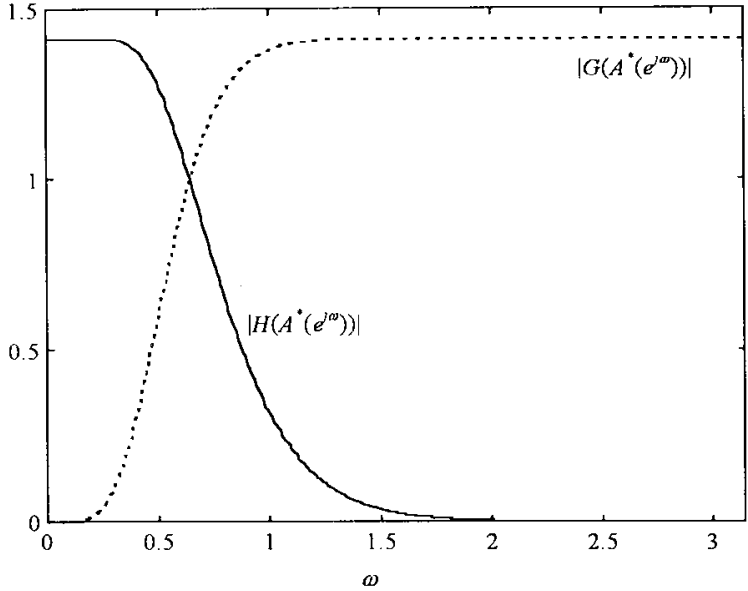

Fig. 2. Frequency warped QMF transfer functions.

The sequences $\varphi_{1, m}(k)$ and $\psi_{1, m}(k)$ constitute the sets associated with the frequency warping filterbank and play the same role as that of $h_{m}(k)$ and $g_{m}(k)$ in ordinary PR filterbanks. Due to the orthogonality and PR property of the filterbank and the orthogonality and completeness of the discrete Laguerre set, the set obtained by adjoining $\varphi_{1, m}(k)$ to $\psi_{1, m}(k)$ is orthogonal and complete in $\ell^{2}(N)$.

The sequences in the new basis are frequency warped and filtered versions of the sequences $h_{m}(k)$ and $g_{m}(k)$. In fact, exploiting (41) of the Laguerre sequences (see the Appendix), it is easy to show that their $z$-transforms are related as

$$
\Phi_{1, m}(z)=\Lambda_{2 m}(z) H\left[A(z)^{-1}\right]=\Lambda_{0}(z) H_{m}\left[A(z)^{-1}\right]
$$

and

$$
\Psi_{1, m}(z)=\Lambda_{2 m}(z) G\left[A(z)^{-1}\right]=\Lambda_{0}(z) G_{m}\left[A(z)^{-1}\right]
$$

where $A(z)$ is the transfer function of a real allpass filter with single pole in $z=b, b$ being the Laguerre parameter. The phase of $A(z)$ induces a warping of the frequency axis given, for real values of $b$, by the map

$$
\theta(\omega)=-\arg A\left(e^{j \omega}\right)=\omega+2 \tan ^{-1}\left(\frac{b \sin \omega}{1-b \cos \omega}\right) .
$$

This map is the unique one-to-one frequency warping map that can be realized by means of rational transfer functions [11]. It shifts the $\pi / 2$ cut-off frequency of the ordinary filterbank to the frequency $\pi / 2-2 \tan ^{-1}(b)$. The bandwidths of the warped filters are complementary with respect to $\pi$ and can be arbitrarily designed by choosing the Laguerre parameter, as shown in Fig. 2. This important property allows us to break 
the half-bandwidth barrier of the ordinary QMF filterbanks while preserving the orthogonality and completeness of the associated set.

The transfer function $\Lambda_{0}(z)$, which is needed for orthogonality, corresponds to the zero-order Laguerre sequence and is lowpass or highpass according to whether $b$ is positive or negative. This filter envelopes the magnitude responses of the QMF pair. Furthermore, since $\Lambda_{m}(z)=\Lambda_{0}(z) A(z)^{m}$, it is apparent from (1) and (2) that $\varphi_{1, m}(k)$ and $\psi_{1, m}(k)$ are obtained by allpass filtering rather than shifting, respectively, $\varphi_{1, m-1}(k)$ and $\psi_{1, m-1}(k)$, i.e.,

$$
\Phi_{1, m}(z)=A(z)^{2} \Phi_{1, m-1}(z)
$$

and

$$
\Psi_{1, m}(z)=A(z)^{2} \Psi_{1, m-1}(z)
$$

Thus, the shape of the warped sequences changes with the index $m$ while their magnitude Fourier transform is preserved.

To conclude this section, we note that in the special case of Haar QMF's $H(z)=\left(1+z^{-1}\right) / \sqrt{2}$ and $G(z)=(1-$ $\left.z^{-1}\right) / \sqrt{2}$, the set associated with the warped filterbank coincides with a particular realization of Kautz sequences [14] with real parameter equal to $b$. The general class of sets associated with the warped filterbanks extends the class of singleparameter Kautz sequences, allowing for arbitrary QMF's. On the other hand, there is a large class of multiparameter Kautz sequences that cannot be implemented in the warped filterbank, not even if we based our construction on multiparameter Laguerre sequences.

The results illustrated in this section are easily extended to $M$-band filterbanks and to the biorthogonal case in which the analysis and synthesis impulse responses are distinct.

\section{ITERATED WARPED FILTERBANKS AND DISCRETE-TIME WARPED WAVELETS}

Following the same construction of ordinary discrete-time wavelets, one can build frequency warped wavelets by iterating the frequency warped filterbank described in the previous section. At each step, the lowpass branch is cut, and a new frequency warped filterbank is inserted. This amounts to projecting the analysis coefficients of the lowpass branch over the set associated with the filterbank. To add flexibility, one may choose a different Laguerre parameter at each stage. It is therefore convenient to introduce the following notation. Let

$$
h_{n, m}(k) \equiv \sum_{r=0}^{\infty} \lambda_{n, r}(k) h_{m}(r)
$$

and

$$
g_{n, m}(k) \equiv \sum_{r=0}^{\infty} \lambda_{n, r}(k) g_{m}(r)
$$

denote the $n$ th-stage projection set, where $\lambda_{n, r}(k)$ is the order $r$ Laguerre sequence with parameter $b_{n}$. In particular, we have $h_{1, m}(k)=\varphi_{1, m}(k)$ and $g_{1, m}(k)=\psi_{1, m}(k)$. Let $v_{n, m}$ and $w_{n, m}$ denote the $n$ th-stage projection coefficients of the lowpass and highpass branch, respectively. We have the iteration

$$
v_{n, m}=\sum_{r=0}^{\infty} h_{n, m}(r) v_{n-1, r}
$$

and

$$
w_{n, m}=\sum_{r=0}^{\infty} g_{n, m}(r) v_{n-1, r}
$$

On the other hand, for any arbitrary signal $x(k)$, the coefficients $v_{n, m}$ are obtained through projection over the set of frequency warped scaling sequences, i.e.,

$$
v_{n, m}=\sum_{k=0}^{\infty} x(k) \varphi_{n, m}(k)
$$

Substituting (10) in both sides of (8) and (9) and interchanging the order of summation, in view of the arbitrariness of the signal, we obtain the following recurrence.

$$
\varphi_{n, m}(k)=\sum_{r=0}^{\infty} h_{n, m}(r) \varphi_{n-1, r}(k)
$$

and

$$
\psi_{n, m}(k)=\sum_{r=0}^{\infty} g_{n, m}(r) \varphi_{n-1, r}(k)
$$

We will show that the $z$ transforms of the warped scaling sequences and wavelets, respectively, have the form

$$
\Phi_{n, m}(z)=B_{n}(z)^{2 m} \Phi_{n, 0}(z)
$$

and

$$
\Psi_{n, m}(z)=B_{n}(z)^{2 m} \Psi_{n, 0}(z)
$$

where

$$
B_{n}(z)=A_{n}\left[B_{n-1}(z)^{-2}\right]=A_{n}\left\{A_{n-1}\left[\cdots A_{1}(z)^{-2} \cdots\right]^{-2}\right\}
$$

with $B_{0}(z)=z^{-1 / 2}$, and $A_{n}(z)$ is the $n$ th-stage first-order allpass with parameter $b_{n}$.

Furthermore

$$
\Phi_{n, 0}(z)=\prod_{k=1}^{n}\left\{\Lambda_{k, 0}\left[B_{k-1}(z)^{-2}\right] H\left[B_{k}(z)^{-1}\right]\right\}
$$

and

$$
\Psi_{n, 0}(z)=\Lambda_{n, 0}\left[B_{n-1}(z)^{-2}\right] G\left[B_{n}(z)^{-1}\right] \Phi_{n-1,0}(z) .
$$

In order to show (13) and (16), notice that from (4), it follows that $\Phi_{1, m}(z)=A_{1}(z)^{2 m} \Phi_{1,0}(z)$. Thus, $\Phi_{1, m}(z)$ has the same form as (13) with $B_{1}(z)=A_{1}(z)^{2}$. In addition, from (1), we observe that $\Phi_{1,0}(z)$ has the same form as (16). Suppose now that both (13) and (16) are verified up to $n=s-1$. 
From (11), it follows that

$$
\begin{aligned}
\Phi_{s, m}(z) & =\sum_{r=0}^{\infty} h_{s, m}(r) B_{s-1}(z)^{2 r} \Phi_{s-1,0}(z) \\
& =H_{s, m}\left[B_{s-1}(z)^{-2}\right] \Phi_{s-1,0}(z) .
\end{aligned}
$$

On the other hand, from (6), we have

$$
H_{s, m}(z)=A_{s}(z)^{2 m} \Lambda_{s, 0}(z) H\left[A_{s}(z)^{-1}\right]
$$

and therefore

$$
\begin{aligned}
\Phi_{s, m}(z)= & A_{s}\left[B_{s-1}(z)^{-2}\right]^{2 m} \Lambda_{s, 0}\left[B_{s-1}(z)^{-2}\right] \\
& \cdot H\left\{A_{s}\left[B_{s-1}(z)^{-2}\right]^{-1}\right\} \Phi_{s-1,0}(z) .
\end{aligned}
$$

Thus, using (15)

$$
\begin{aligned}
\Phi_{s, m}(z) & =B_{s}(z)^{2 m} \Lambda_{s, 0}\left[B_{s-1}(z)^{-2}\right] H\left[B_{s}(z)^{-1}\right] \Phi_{s-1,0}(z) \\
& =B_{s}(z)^{2 m} \Phi_{s, 0}(z)
\end{aligned}
$$

and hence, both (13) and (16) are valid for $n=s$. By induction, they are valid for any $n$. In a similar fashion, we can prove (14) and (17).

The allpass $B_{n}(z)$ determines the total amount of warping imparted to the nth-stage QMF's. The corresponding frequency map obtained from the phase of the $B_{n}(z)$ is given by the composite function

$$
\Omega_{n}(\omega)=\theta_{n}\left(2 \theta_{n-1}\left\{\cdots 2 \theta_{2}\left[2 \theta_{1}(\omega)\right] \cdots\right\}\right)
$$

where each $\theta_{k}(\omega)$ has the form (3), with parameter $b_{k}$. The factor of 2 in (18) derives from the downsampling-by-2 operation performed in the Laguerre transform domain (see Fig. 1). Of course, at each iteration, one should consider the phase $\Omega_{k}(\omega)$ as congruent modulo $2 \pi$. However, the result is not changed if we consider the unwarped phase and then apply the congruence at the last step if needed. Defining $\Omega_{0} \equiv \omega / 2$, one can write

$$
\Omega_{n}(\omega)=\theta_{n}\left[2 \Omega_{n-1}(\omega)\right] .
$$

By selecting the parameters $b_{k}$, the cut-off frequencies $\omega_{k}$ of the warped filters may be chosen at will, provided that the condition $\omega_{1}>\omega_{2}>\cdots>\omega_{N}$ is fulfilled. Since the cutoff frequency of the ordinary QMF's occurs at $\omega=\pi / 2$, the cut-off frequencies of the warped filters will occur, for any $n$, at the smallest positive root of the equation $\Omega_{n}(\omega)=\pi / 2$. The task of determining these roots is highly simplified if we observe that the inverse map $\theta^{-1}(\omega)$ is obtained from (3) by reversing the sign of the parameter $b$. Thus, $\omega_{1}=\theta_{1}^{-1}(\pi / 2)=$ $\pi / 2-2 \tan ^{-1}\left(b_{1}\right)$, which yields $b_{1}=\tan \left[\left(\pi-2 \omega_{1}\right) / 4\right]$. Once we know $b_{1}$, we can determine $b_{2}$ from the equality $2 \theta_{1}\left(\varpi_{2}\right)=\theta_{2}^{-1}(\pi / 2)=\pi / 2-2 \tan ^{-1}\left(b_{2}\right)$ from which $b_{2}=\tan \left[\pi / 4-\theta_{1}\left(\omega_{2}\right)\right]$. More generally, since

$$
2 \Omega_{n-1}\left(\omega_{n}\right)=\theta_{n}^{-1}(\pi / 2)=\frac{\pi}{2}-2 \tan ^{-1}\left(b_{n}\right)
$$

we have

$$
b_{n}=\tan \left[\frac{\pi}{4}-\Omega_{n-1}\left(\omega_{n}\right)\right] .
$$

The choice of the Laguerre parameters depends on the application. The frequency warped wavelets can be adapted

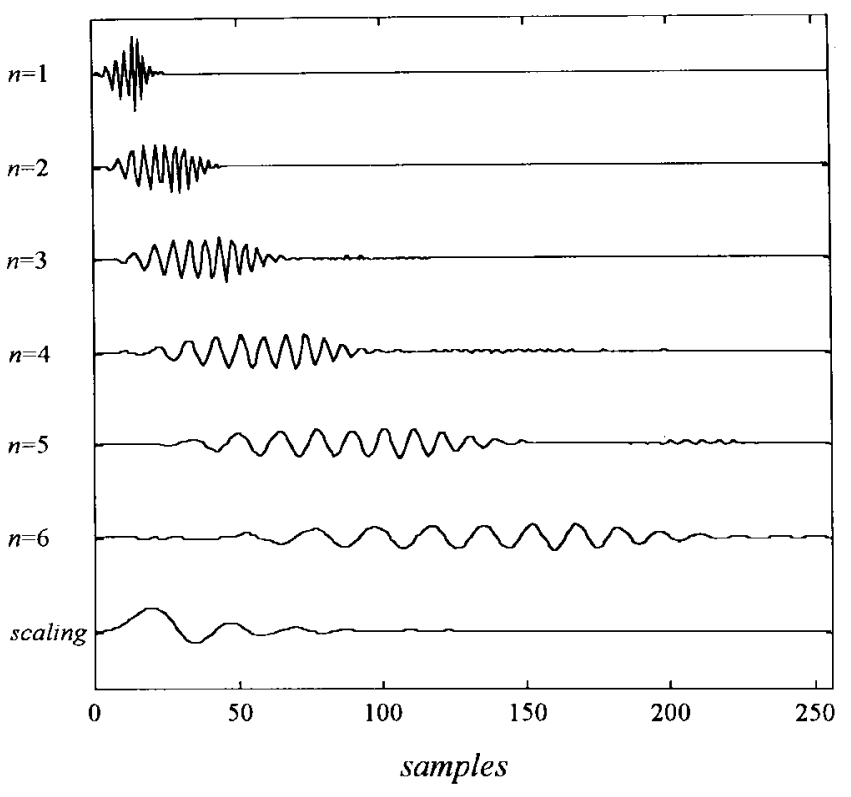

(a)

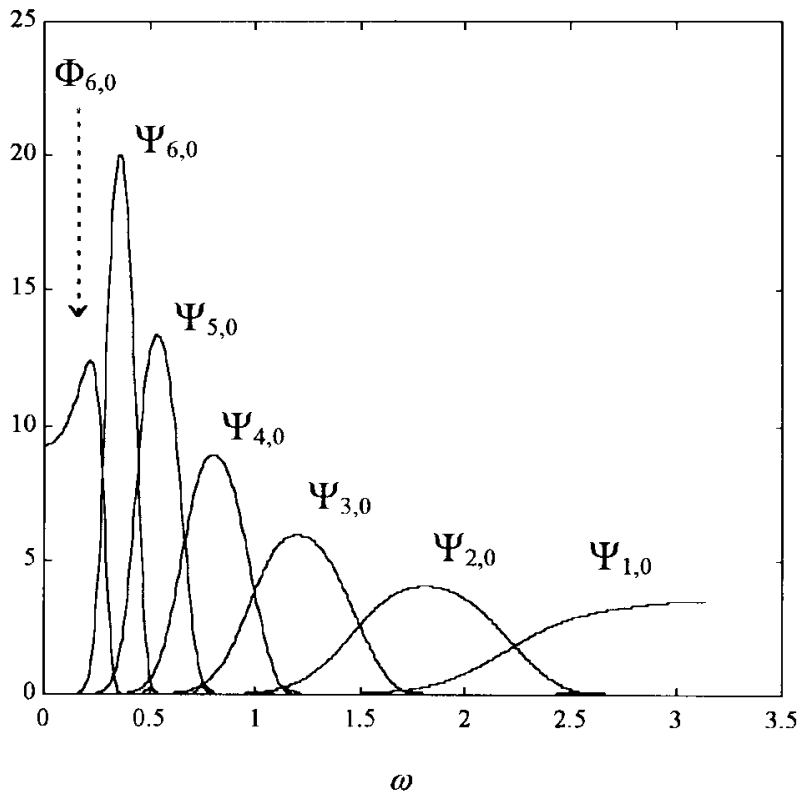

(b)

Fig. 3. Warped wavelets with exponential cutoff $a=\frac{2}{3}$. (a) Time domain. (b) Frequency domain.

to signal features, e.g., in order to separate them by projection on distinct basis sequences. A particular choice of cut-off frequency may be derived from the structure of classical wavelet transforms, i.e., we may select $\omega_{n}=\pi a^{n}$, where $a<1$. In this way, one can fit rational or transcendental bandwidths into the dyadic scheme. In Fig. 3, a set of discretetime frequency warped wavelets with $a=2 / 3$ is shown. The warped wavelets provide an unconventional tiling of the time-frequency plane by means of curvilinear cells whose area is constant, which is shown in Fig. 4. This is due to the frequency-dependent phase delay that makes the localization of the wavelets depend on frequency as well.

It must be pointed out that the described warped wavelets can be implemented by means of rational transfer functions. 


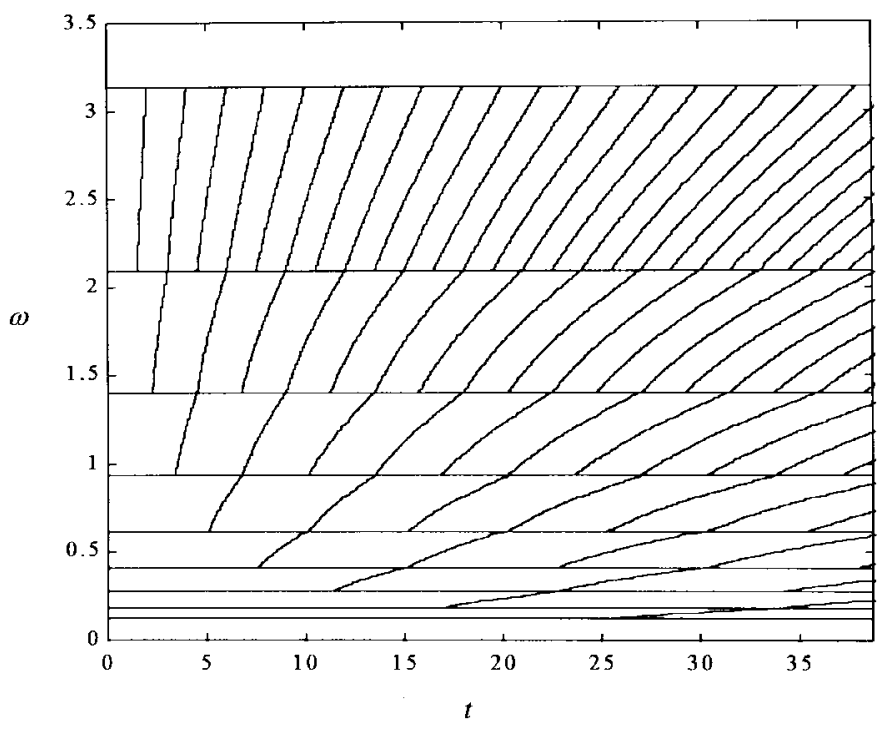

(a)

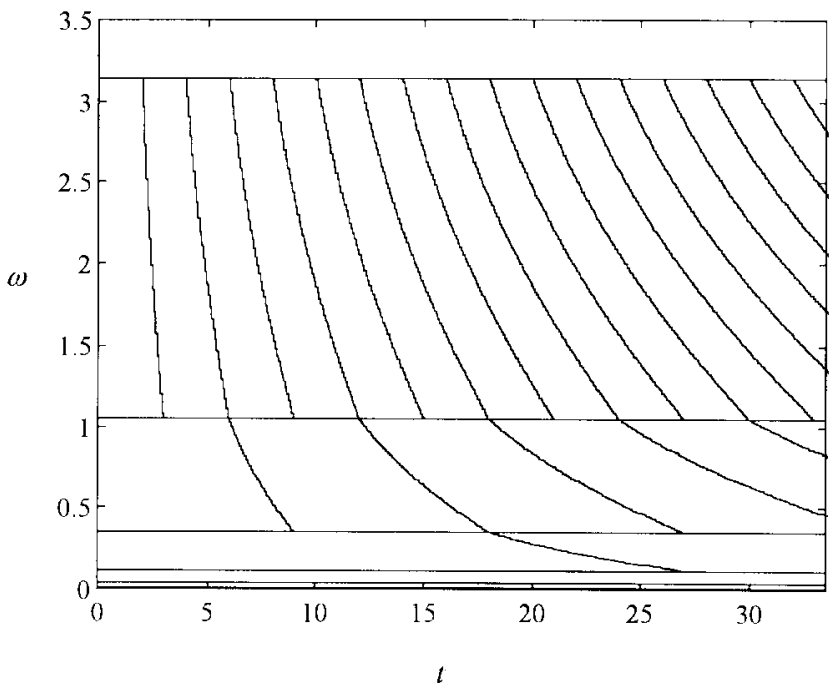

(b)

Fig. 4. Tiling the time-frequency plane with warped wavelets. (a) With parameter $a=\frac{2}{3}$. (b) With parameter $a=\frac{1}{3}$.

Since each individual warping map is the unique one-to-one frequency warping map that can be realized by means of rational transfer functions, our scheme provides the unique way of implementing warped wavelet expansion in digital structures.

\section{Continuous-Time Warped WaVelets}

The question whether infinite iterations of the frequency warped filterbank lead to well-defined continuous-time orthogonal and complete wavelets is a rather challenging mathematical problem. In this section, we will explore this field in the attempt to provide a positive answer. Our results are supported by extensive numerical simulations.

Our starting point is the iterated map (18). Each elementary map is a differentiable, increasing function $\theta_{k}(\omega)$ mapping the interval $[-\pi, \pi)$ onto itself. If $b_{k}<0$, then $\theta_{k}(\omega)$ is convex for $0<\omega<\pi$ and concave for $-\pi<\omega<0$. This happens vice versa if $b_{k}<0$, and then, $\theta_{k}(\omega)$ is concave for $0<\omega<\pi$ and convex for $-\pi<\omega<0$. The points $\varpi_{r}=r \pi, r \in Z$ are the fixed points of each of the maps. However, since at each iteration we double $\theta_{k}(\omega)$ before applying $\theta_{k+1}(\omega)$, the only surviving fixed point is $\varpi_{0}=0$.

The iterated map $\Omega_{n}(\omega)$ given in (18) is an increasing function mapping the interval $[-\pi, \pi)$ one-to-one and onto $\left[-2^{n-1} \pi, 2^{n-1} \pi\right)$. It embeds both warping and upsampling. In considering conversions from digital to analog frequency, it is convenient to extend the definition of the iterated map over the entire real axis while keeping the same functional form and symbol. The extended map is monotone and equal to a linear trend $2^{n-1} \omega$ plus a periodic function. Its inverse is

$$
\Omega_{n}^{-1}(\omega)=\theta_{1}^{-1}\left\{\frac{1}{2} \theta_{2}^{-1}\left[\cdots \frac{1}{2} \theta_{n}^{-1}(\omega) \cdots\right]\right\}
$$

with $\Omega_{0}^{-1}(\omega)=2 \omega$, where $\theta_{k}^{-1}(\omega)$ is obtained from $\theta_{k}(\omega)$ by changing the sign to the parameter $b_{k}$. The map $\Omega_{n}^{-1}(\omega)$ maps $\left[-2^{n-1} \pi, 2^{n-1} \pi\right)$ one-to-one and onto $[-\pi, \pi)$ and embeds warping and down scaling.

The DFT of the scaling sequence may be written in terms of the frequency map as

$$
\Phi_{N, 0}\left(e^{j \omega}\right)=\prod_{k=1}^{N}\left\{\Lambda_{k, 0}\left[e^{j 2 \Omega_{k-1}(\omega)}\right] H\left[e^{j \Omega_{k}(\omega)}\right]\right\} .
$$

We are interested in extending the construction of ordinary continuous-time wavelets to the iterated warping case. When we let $n$ go to infinity, the characteristics of the iterated warping map depend on the choice of the parameter sequence $b_{k}$ related to the choice of the wavelet cut-off frequencies via (19). Convergence modulo $2 \pi$ of the iterated map may be checked by studying the behavior of the poles of the order $2^{n}$ allpass filter $B_{n}(z)$ of which $\Omega_{n}(\omega)$ represents the phase. A necessary and sufficient condition [1] for the infinite Blaschke product $B_{\infty}(z)$, with poles in $z=\varsigma_{k}, k \in N$, to converge uniformly on compact subsets is that $\sum_{k}\left(1-\left|\varsigma_{k}\right|\right)<\infty$. We will consider two special cases where the $b_{k}$ 's are either constant or are determined by the exponential cut-off choice $\omega_{n}=\pi a^{n}$.

The constant $b_{k}=b$ case is the easiest to handle since the ordering of the elementary maps is irrelevant, and we may drop their indexes. The resulting cut-off frequencies satisfy the following iteration: $\omega_{n+1}=\theta^{-1}\left(\omega_{n} / 2\right)$, with $\omega_{1}=\pi / 2-$ $2 \tan ^{-1}(b)$. If $b<-\frac{1}{3}$, this iteration converges to a stable fixed point located in $\omega=2 \tan ^{-1} \sqrt{(-3 b-1) /(1-b)}$. This range of values must be avoided since the cut-off frequencies cluster around the fixed point rather than around the origin. For $b>-1 / 3$, it can be seen that the cut-off frequencies behave asymptotically like a decreasing exponential with decay rate dependent on $b$.

In the ordinary wavelet case, the digital frequency is obtained by rescaling the analog frequency. A suitable candidate in the warped case is given by the inverse map $\Omega_{n}^{-1}(\omega / 2)$, which maps the interval $\left[-2^{n} \pi, 2^{n} \pi\right)$ onto $[-\pi, \pi)$. Accordingly, we define the $N$ th scaling function approximant as

$$
\tilde{\Phi}^{(N)}(\omega) \equiv C^{N} \Phi_{N, 0}\left[e^{j \Omega_{N}^{-1}(\omega / 2)}\right]
$$


where $\omega \in\left[-2^{n} \pi, 2^{n} \pi\right)$, and $C$ is a constant to be determined. By substituting the product (21) in (22), we obtain

$$
\begin{aligned}
& \tilde{\Phi}^{(N)}(\omega)= \\
& \prod_{k=1}^{N}\left(C \Lambda_{k, 0}\left\{e^{j 2 \Omega_{k-1}\left[\Omega_{N}^{-1}(\omega / 2)\right]}\right\} H\left\{e^{j \Omega_{k}\left[\Omega_{N}^{-1}(\omega / 2)\right]}\right\}\right) .
\end{aligned}
$$

The arguments of $\Lambda_{k, 0}=\Lambda_{0}$ and $H$ have the form

$$
\Omega_{k}\left[\Omega_{N}^{-1}(\omega)\right]=2^{-1} \Omega_{N-k}(\omega) .
$$

We remark that the previous identity is valid only in the constant $b_{k}$ case. Exploiting (24) and changing the index in the product (21) to $l=N-k$, we obtain

$$
\tilde{\Phi}^{(N)}(\omega)=\prod_{l=0}^{N-1}\left\{C \Lambda_{0}\left[e^{j \Omega_{l+1}^{-1}(\omega / 2)}\right] H\left[e^{j 2^{-1} \Omega_{l}^{-1}(\omega / 2)}\right]\right\} .
$$

Finally, we define the scaling function as the limit of the $N$ th scaling function approximant

$$
\tilde{\Phi}(\omega) \equiv \lim _{N} \tilde{\Phi}^{(N)}(\omega)
$$

if the infinite product (25) converges.

A necessary condition for the product to converge is that its factors tend to 1 as $l$ increases. The convergence depends both on the parameter sequence $b_{k}$ and the transfer function $H$ and should be checked separately in each case.

As $l$ grows, $\Omega_{l}^{-1}(\omega)$ maps points in any neighborhood of 0 to points in an increasingly narrower neighborhood of 0 . Therefore, the constant $C$ can be determined from the condition

$$
\frac{1}{C}=\Lambda_{0}(1) H(1)=\sqrt{2 \frac{1+b}{1-b}} .
$$

With this position, the scaling function is completely determined and is given, in the frequency domain, by the infinite product

$\tilde{\Phi}(\omega)=\prod_{l=0}^{\infty}\left\{\sqrt{\frac{1-b}{2(1+b)}} \Lambda_{0}\left[e^{j \Omega_{l+1}^{-1}(\omega / 2)}\right] H\left[e^{j 2^{-1} \Omega_{l}^{-1}(\omega / 2)}\right]\right\}$.

Examples of warped scaling functions with constant $b$ are shown in Fig. 5. We remark that the wavelets generated using negative parameters are less regular than those generated using positive parameters. This is due to the fact that when $b<0$, the order zero Laguerre term $\Lambda_{0}(z)$ becomes highpass and has a tendency to absorb regularity from the lowpass filter $H(z)$. Indeed, it appears from the figure that the negative $b$ scaling functions have fractal-like behavior. Conversely, the positive $b$ scaling functions are smoother than regular wavelets due to the lowpass Laguerre term $\Lambda_{0}(z)$.

Exploiting the relationship

$$
\Omega_{l}^{-1}\left[\Omega_{1}(\omega)\right]=\Omega_{l-1}^{-1}(\omega / 2)
$$

which is valid in the constant $b_{k}$ case, one can obtain from (28) the warped form of the two-scale equation

$$
\tilde{\Phi}\left[2 \Omega_{1}(\omega)\right]=C \Lambda_{0}\left(e^{j \omega}\right) H\left(e^{j \Omega_{1}(\omega)}\right) \tilde{\Phi}(\omega) .
$$

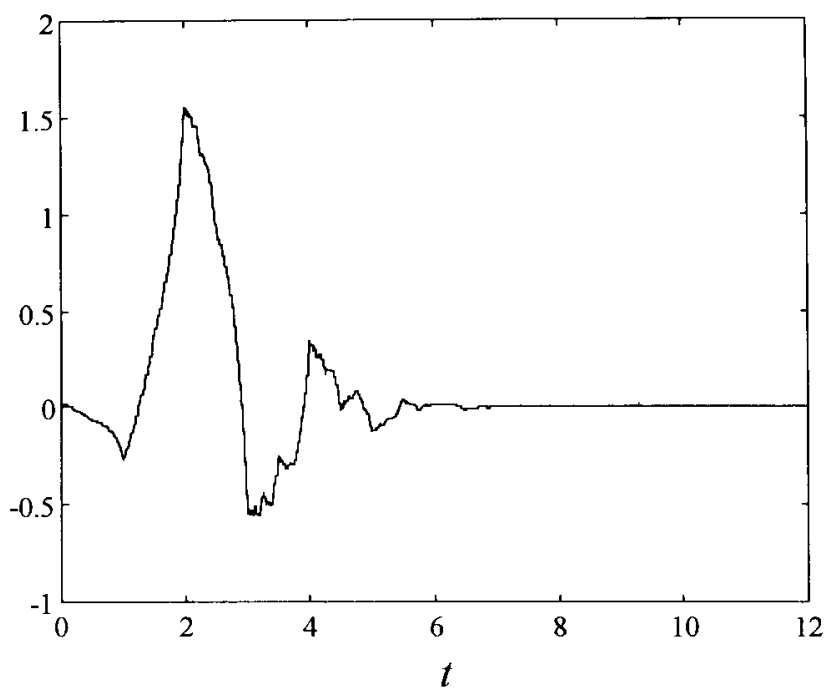

(a)

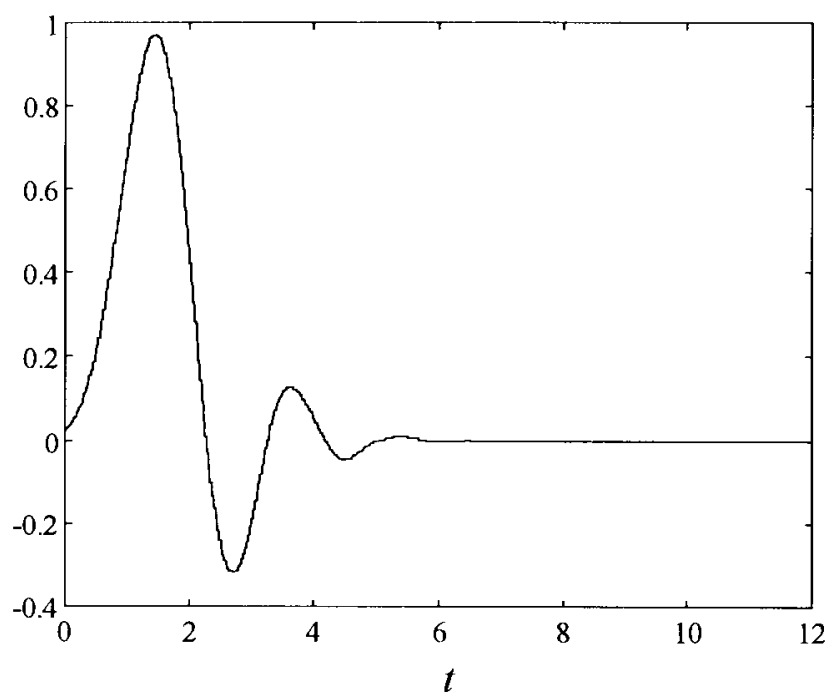

(b)

Fig. 5. Continuous-time warped scaling function. (a) With $b=-0.2$. (b) With $b=0.2$.

Repeated application of (30) leads to the following relationship between discrete- and continuous-time warped scaling functions.

$$
\tilde{\Phi}\left[2 \Omega_{n}(\omega)\right]=C^{n} \Phi_{n, 0}(\omega) \tilde{\Phi}(\omega) .
$$

Equation (31) gives the level- $n$ scaling function $\tilde{\Phi}_{n, 0}(\omega)=$ $C^{-n} \tilde{\Phi}\left[2 \Omega_{n}(\omega)\right]$ in terms of the level 0 scaling function $\tilde{\Phi}(\omega)$. Accordingly, the continuous-time wavelets are given by

$$
\tilde{\Psi}_{n, 0}(\omega)=\Psi_{n, 0}(\omega) \tilde{\Phi}(\omega) .
$$

As for the discrete-time warped wavelets, the continuous-time warped wavelets are not translation invariant. Since, from (13), the delay term corresponding to the $m$ th translate of the level $N$ discrete wavelet is $e^{-j 2 m \Omega_{N}(\omega)}$, substituting, just as in (22), the digital frequency $\omega$ with the map $\Omega_{N}^{-1}(\omega / 2)$, we show that the level 0 continuous-time warped scaling functions are obtained by simple translation

$$
\tilde{\Phi}_{0, m}(\omega)=e^{-j m \omega} \tilde{\Phi}_{0,0}(\omega) .
$$


However, at higher scales, we have

$$
\tilde{\Phi}_{n, m}(\omega)=e^{-j 2 m \Omega_{n}(\omega)} \tilde{\Phi}_{n, 0}(\omega) .
$$

The frequency-dependent delay term alters the shape of the scaling functions and wavelet translates.

Following a reasoning similar to that presented in [18] for the ordinary wavelets, by exploiting the orthogonality and completeness of the discrete-time wavelets, one is able to show that if the product (28) converges pointwise, the continuoustime wavelet set is orthogonal and complete in $L^{2}(R)$.

Due to the asymptotically exponential behavior, the exponential cut-off choice $\omega_{n}=\pi a^{n}$ may be well approximated by the constant $b$ warping. However, its anomalous behavior for $b \leq-\frac{1}{3}$ prevents the use of the constant $b$ warping for obtaining high resolution at high frequencies (small scale index), say, with $a>\frac{4}{5}$. It is possible to show that the sequence of parameters $b_{k}$ determined by the exponential cut-off choice satisfies the following properties:

1) all $b_{k}$ have the same sign, resulting in $b_{k}>0$ if $0<a<\frac{1}{2}$ and $b_{k}<0$ if $\frac{1}{2}<a<1$.

2) The sequence of parameters converges exponentially to the limit value $b=(1-2 a) /(1+2 a)$.

Since (24) is not valid in this case, the scaling function approximant will keep the general form (23). However, due to the fast convergence of the sequence of parameters-essential convergence is attained in less than 10 steps-(24) is valid asymptotically, and it is always possible to split the product (23) into two products. The product corresponding to large values of the index behaves approximately as in the constant $b$ case. The constant $C$ may be derived from (27) by replacing $b$ with its asymptotic value, which gives $C=\sqrt{a}$.

Our numeric experiments over the allowed range of the scaling factor $a$ show that the map $\Omega_{n}^{\bullet}(\omega) \equiv \Omega_{n}(\omega) / 2^{n-1}$ quickly converges to an approximately selfsimilar function $\Omega^{\bullet}(\omega)$. A family of maps $\Omega^{\bullet}(\omega)$ is plotted in Fig. 6 for the entire range of the parameter $a$. In order to better appreciate the fractal behavior of the asymptotic map, in Fig. 7, we plot a member of the family with the linear trend $\omega$ subtracted. Similarly, $\Omega_{n}^{\square}(\omega) \equiv \Omega_{n}^{-1}\left(2^{n-1} \omega\right)$ converges to a fractal function that is the inverse of $\Omega^{\bullet}(\omega)$. As $n$ grows, the poles of the allpass $B_{n}(z)$ quickly move toward the unit circle, as illustrated in Fig. 8. We argue that the condition for the convergence of the Blaschke product is fulfilled in our case.

The function $\Omega^{\bullet}(\omega)$ satisfies the selfsimilarity property

$2^{k} \Omega^{\bullet}\left(a^{k} \omega\right) \approx \Omega^{\bullet}(\omega), \quad$ for any integer $k,-\pi \leq \omega \leq \pi$.

In other words $\Omega^{\bullet}(\omega)$ behaves much like an $a$-homogeneous function in the sense given in [27], i.e., $\Omega^{\bullet}(\omega) \approx$ $a^{-k H} \Omega^{\bullet}\left(a^{k} \omega\right)$, with degree $H=\log _{a} \frac{1}{2}$. However, the selfsimilarity relationship holds only approximately true, although selfsimilarity is much more accurate within the exponential cut-off choice than in the uniform $b$ case. We found that for large $n, \Omega_{n}^{\bullet}(\omega)$ satisfies (33) within an error of the order of $10^{-3}$ for $k=1$. The error quickly drops below machine precision for $20<k<n / 2$, after which, the error starts to increase again.

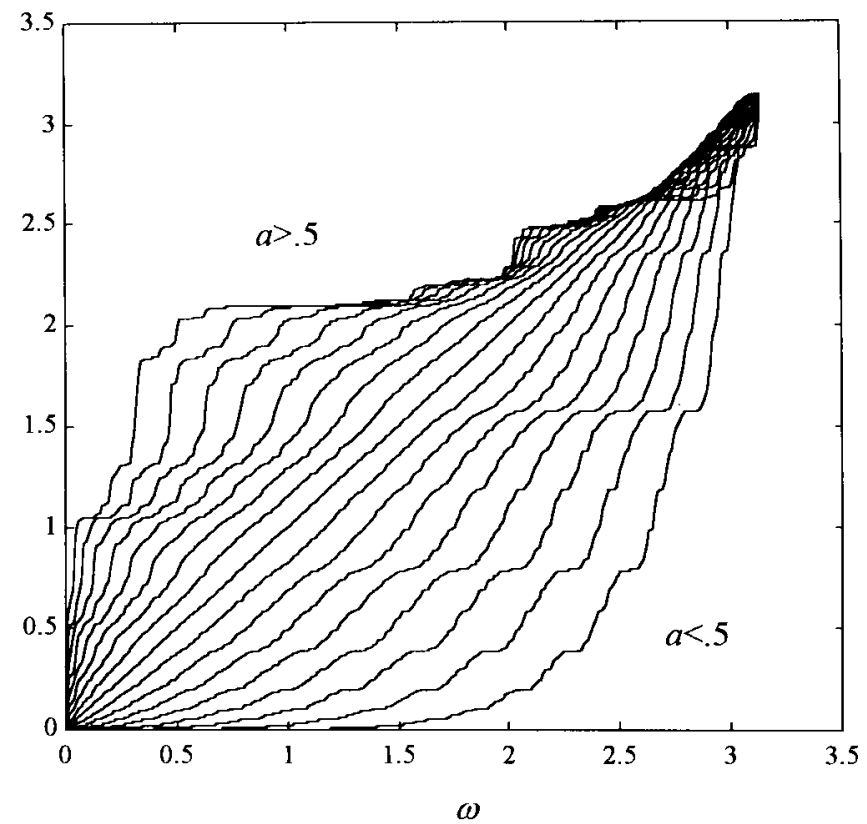

Fig. 6. Family of iterated warping maps $\Omega^{\bullet}(\omega)$ with exponential cutoff.

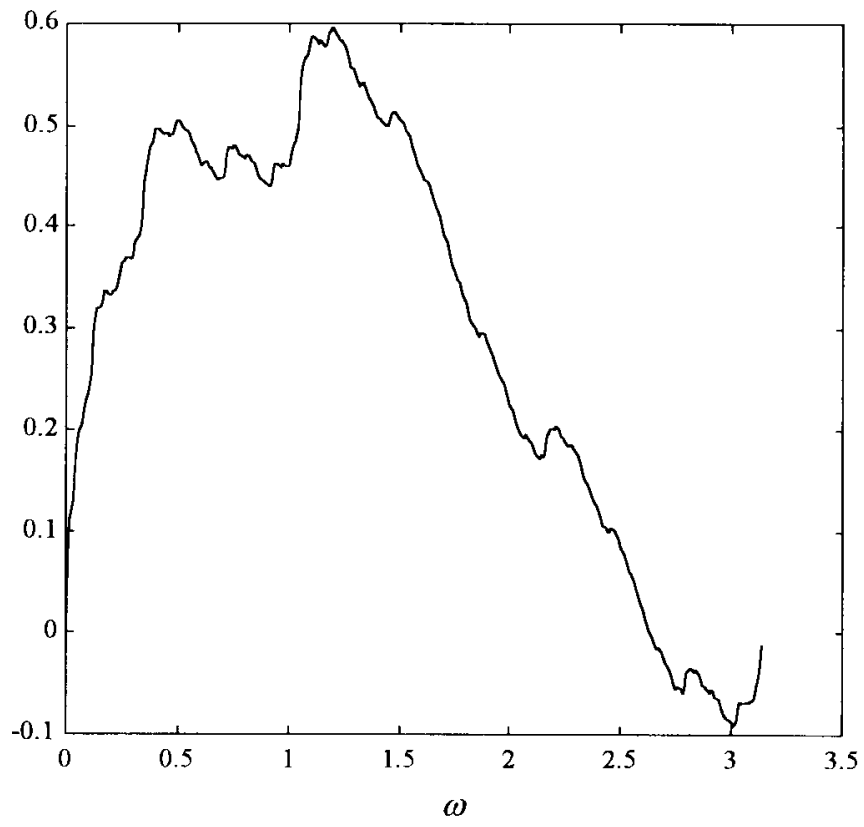

Fig. 7. Iterated warping map ( $a=\frac{1}{3}$ ) with its linear trend removed.

The map $\Omega^{\bullet}(\omega)$ operates a scale conversion from $1 / 2$ to $a$. In fact, if $F(\omega)$ is the DFT of a sequence, then, with good approximation, $F\left[\Omega^{\bullet}\left(a^{k} \omega\right)\right]=F\left[\Omega^{\bullet}(\omega) / 2^{k}\right]$. For this reason, we term $\Omega^{\bullet}(\omega)$ the scale-conversion function.

Another interesting numerical property of $\Omega_{n}(\omega)$ is that, within $1 \%$, the chain of relationships

$$
\Omega_{n+1}\left(a^{n} \omega\right) \approx \Omega_{n}\left(a^{n-1} \omega\right),-\frac{\pi}{a^{n-1}} \leq \omega \leq \frac{\pi}{a^{n-1}}
$$

are verified. This property is illustrated in Fig. 9, where the functions in (34) are plotted, and a set of bifurcation points appear at $\omega_{n}=\pi / a^{n-1}$. These are exactly the points where the lower indexed curve stops behaving like the higher order 

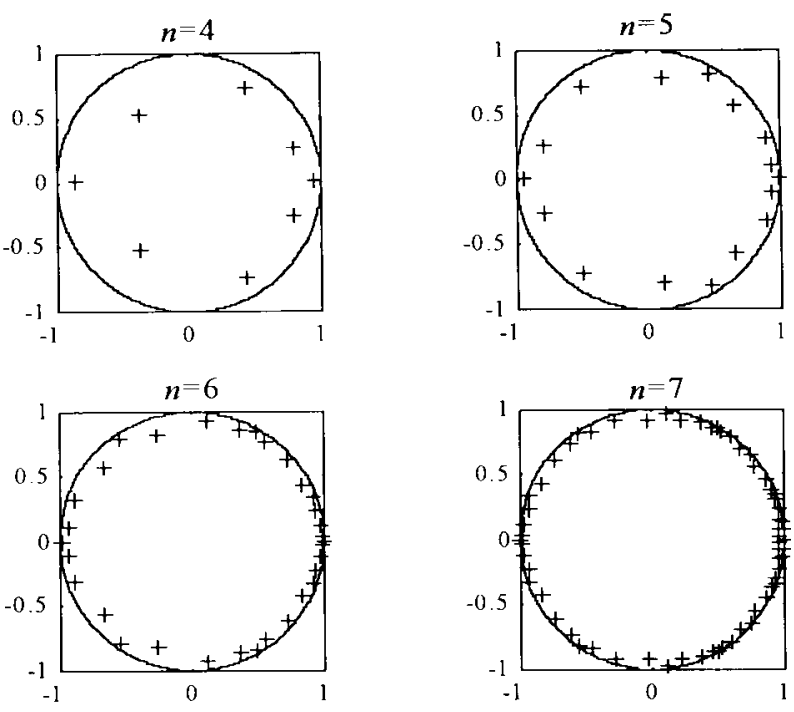

Fig. 8. Progression of poles of the iterated warping allpass filter.

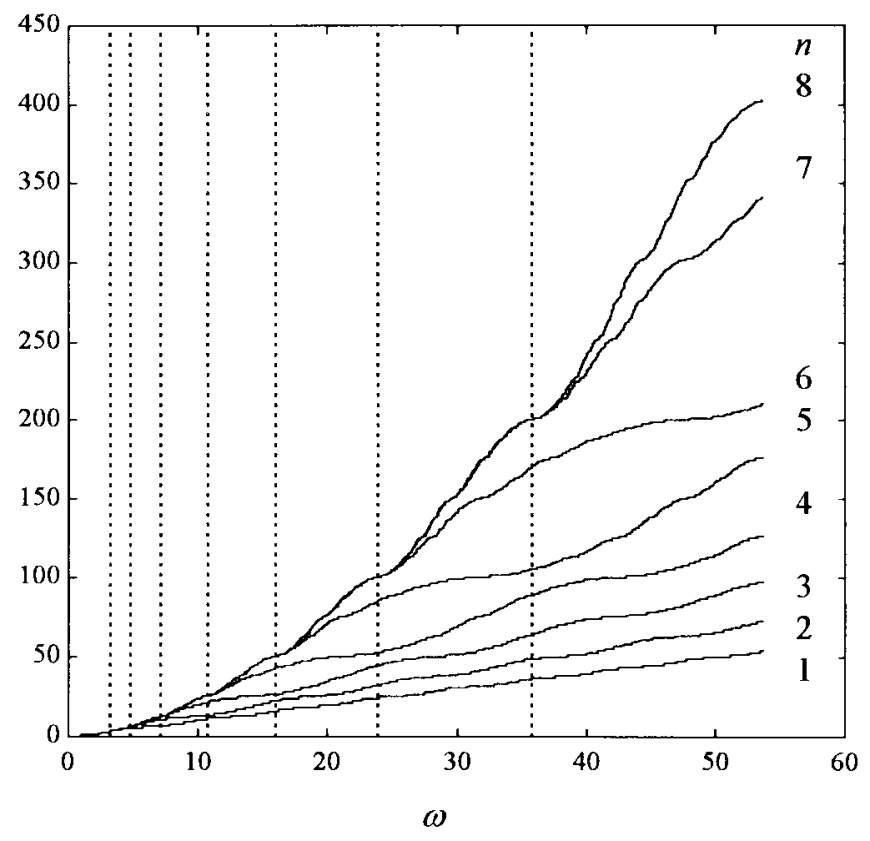

Fig. 9. Plot of the $a$-scaled warping maps $\Omega_{n}\left(a^{n-1} \omega\right) n$, showing bifurcation points.

maps. By exploiting the selfsimilarity of the warping map, it is possible to show that the exponential cut-off wavelets at larger scales are approximately obtained by scaling the wavelets at a smaller scale by a power of $a$.

In concluding this section, we remark that the quality of the exponential cut-off wavelets is similar to that of their constant $b$ counterpart, i.e., the wavelets generated using $a>\frac{1}{2}$ are less regular and more fractal-like than those generated using $a<\frac{1}{2}$.

\section{ApPliCATIONS}

The concept of frequency warping wavelets may be applied in several forms to the analysis and synthesis of signals. Their flexible frequency resolution makes them especially
PSFW-Wavelets : Frequency Domain

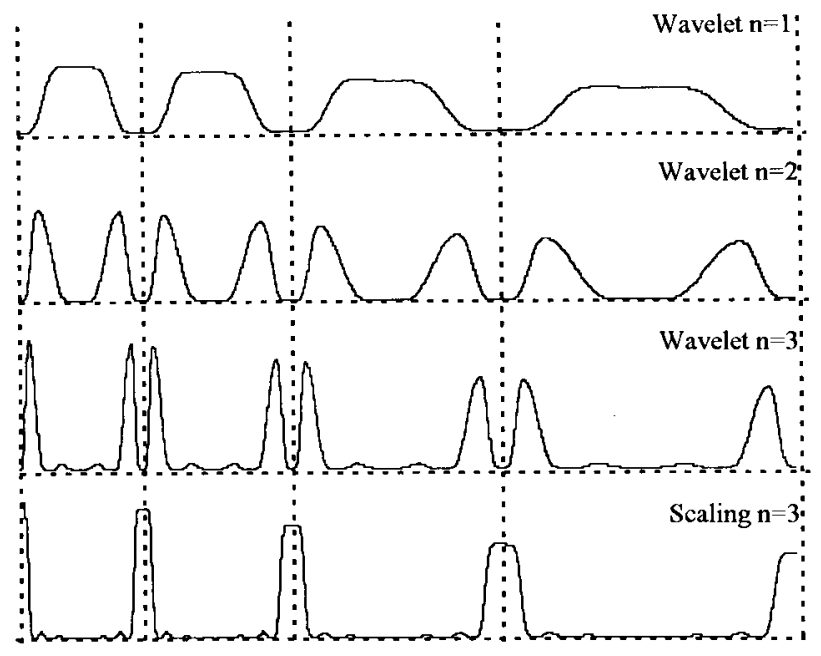

$\omega$

Fig. 10. Frequency spectrum of the PSFW-wavelets and scaling sequence with period $P=3$, and Laguerre parameter $b=0.24$.

useful in those problems where distinct characteristics of the signal lie in nonuniform bands or multibands with prescribed bandwidth. This is particularly desirable in cochlear models and in perceptual representations of speech and hearing.

The drawbacks of frequency warping methods via Laguerre sequences are essentially their computational complexity and the need to truncate the expansion to a finite number of terms. In addition, the number of coefficients required for accurate computation is generally larger than the length of the input signal.

In this section, we describe an application of the frequency warped wavelets to the analysis of inharmonic sounds.

\section{A. Pitch-Synchronous Frequency Warped Wavelets}

An interesting application of warped wavelets is in the analysis and synthesis of pseudo-periodic signals, such as piano tones, rods, and membrane vibrations. Due to stiffness and wave propagation in dispersive media, the peaks in the frequency spectra of these signals are not uniformly spaced. In recent papers, one of the authors defined and applied the pitchsynchronous wavelet transform (PSWT) to the representation of pseudo-periodic sounds [19]-[21]. The main assumption there was that the spacing of the frequency peaks had to be uniform, which is approximately true in voiced speech sounds and in the tones produced by several musical instruments such as the violin, the guitar, and the flute. The analysis of these signals by means of the PSWT presented several advantages in feature extraction, transient detection, and coding. In order to extend the PSWT to the dispersive case, one can prewarp the signal and then apply the PSWT. The scheme is successful if the warping curve compensates inharmonicity. In this section, we define the frequency warped wavelets that implement inharmonicity equalization in orthogonal or biorthogonal expansions and then look at some examples. We shall confine ourselves to the case of multiplexed wavelets 


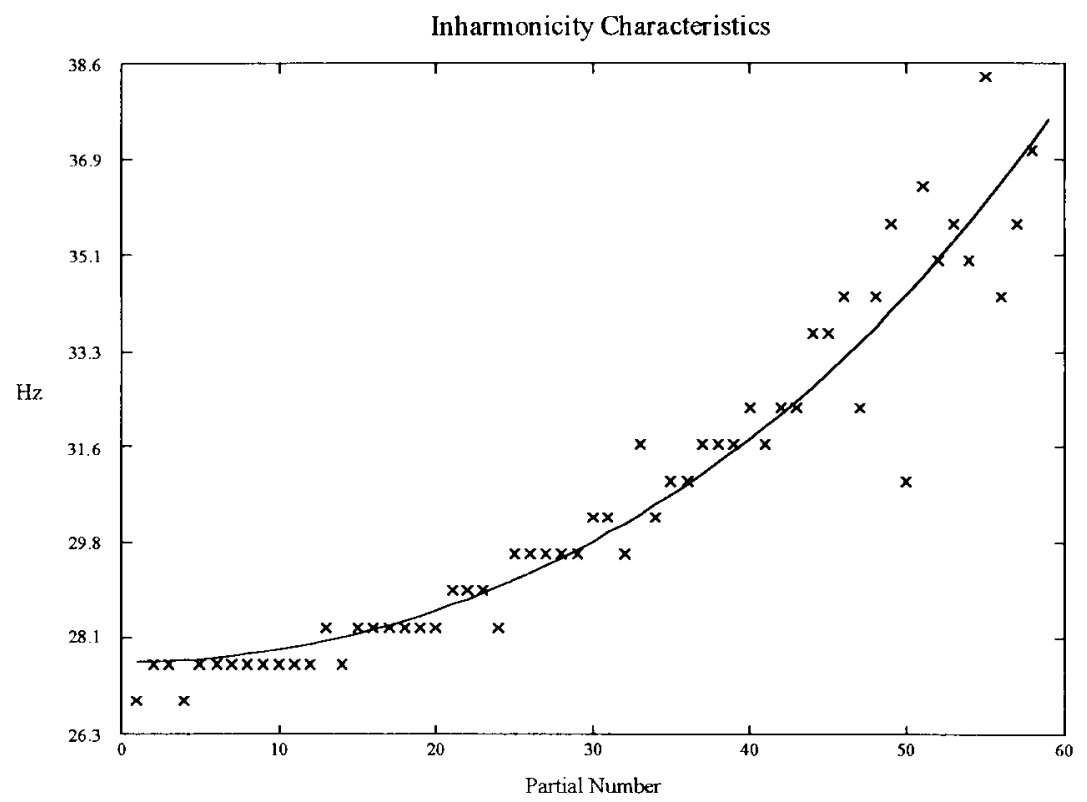

Fig. 11. Inharmonicity characteristics of a piano tone $(27.3 \mathrm{~Hz})$ : difference in $\mathrm{Hz}$ of the frequencies of the first 60 partials and difference of the inverse optimum warping curve $(b=0.4396)$.

obtained from the PS wavelets in the particular case where the pitch $P$ of the signal is constant. The extension of the transform to the time-varying pitch case is straightforward.

In the pitch-synchronous frequency warped wavelet transform (PSFWWT), we project the signal over a discrete Laguerre set, with suitable parameter $b$, and then project the sequence of Laguerre coefficients over a set of PS wavelets. Let

$$
\hat{\xi}_{n, m, q}(k)=\sum_{l} \psi_{n, m}(l) \delta(k-q-l P)
$$

denote the multiplexed wavelets, where $\psi_{n, m}(l)$ are ordinary discrete-time wavelets. We define the PSFW wavelets as

$$
\xi_{n, m, q}(k)=\sum_{l} \psi_{n, m}(l) \lambda_{q+l P}(k) .
$$

Orthogonality and completeness of the PSFW wavelets are inherited from the same properties of both the Laguerre and PS wavelet sets. The expansion coefficients of a signal $s(k)$ over the PSFW wavelets are given by

$$
\begin{aligned}
s_{n, m, q} & =\sum_{k} s(k) \xi_{n, m, q}(k) \\
& =\sum_{r}\left[\sum_{k} s(k) \lambda_{r}(k)\right] \hat{\xi}_{n, m, q}(r) .
\end{aligned}
$$

From the $z$ transform of the PSFW wavelets

$$
\Xi_{n, m, q}(z)=\Lambda_{0}(z) A(z)^{q} \Psi_{n, m}\left[A(z)^{-P}\right]
$$

it is easy to see that their frequency spectra are comblike, consisting of sidebands of nonuniformly spaced pseudoharmonics, as shown in Fig. 10. The individual bandwidth of each of the sidebands depends both on the scale index and on frequency. This is due to the warping of the frequency axis, which modifies both the harmonic frequencies and, locally, the bandwidths. This property is useful for representing inharmonic signals since the more the partials lay apart, the larger the local bandwidths of the scaling sequence and wavelets will be, thus increasing the time resolution.

We experimented the PSFWWT with several piano tones in the lower portion of the keyboard. The purpose of the analysis is to separate transients, such as the hammer and spring noise, from the sound produced by the strings. This is an important problem in sound analysis and synthesis since, e.g., the different characteristics of these components call for distinct synthesis techniques and processing.

The piano strings become quite thick and stiff in the lowpitch region so that dispersion [3] prevents direct application of the PSWT. In order to choose the proper Laguerre warping parameter, we developed an estimation procedure based on a peak-picking algorithm for detecting the center frequencies of the partials of the sound in the frequency domain [2]. This algorithm consists of finding the local maxima of the frequency spectrum using a sliding window. Once the fundamental frequency is detected, we predict the frequency of the next partial. We then suitably scale and translate the window on this basis and detect the frequency of the next local maximum, and so on. This algorithm behaves correctly for frequencies up to about $5 \mathrm{kHz}$ since, in this range, the signal-to-noise ratio of the partials is acceptable. An optimization procedure addresses the problem of fitting the Laguerre warping curve to the inharmonicity data. The frequencies of the partials are matched against the family of Laguerre warping curves, in order to select the parameter $b$ corresponding to the curve that transforms the inharmonic frequencies into the closest-toharmonic series.

In Fig. 11, an optimum fit of inharmonicity data with Laguerre warping characteristics is shown. The inharmonicity data are the differences between subsequent partial frequencies. In the figure, the solid curve represents the difference 


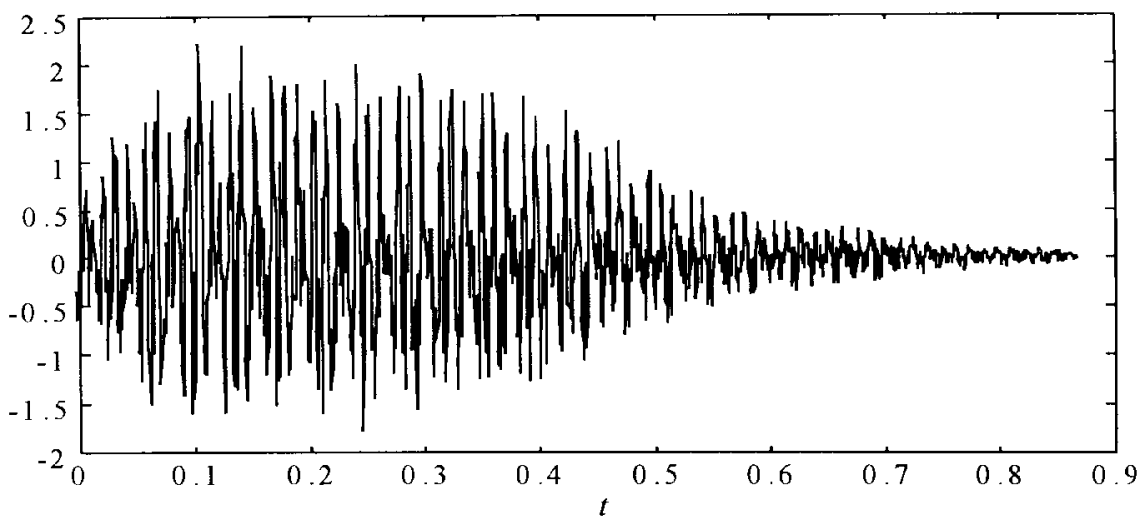

(a)

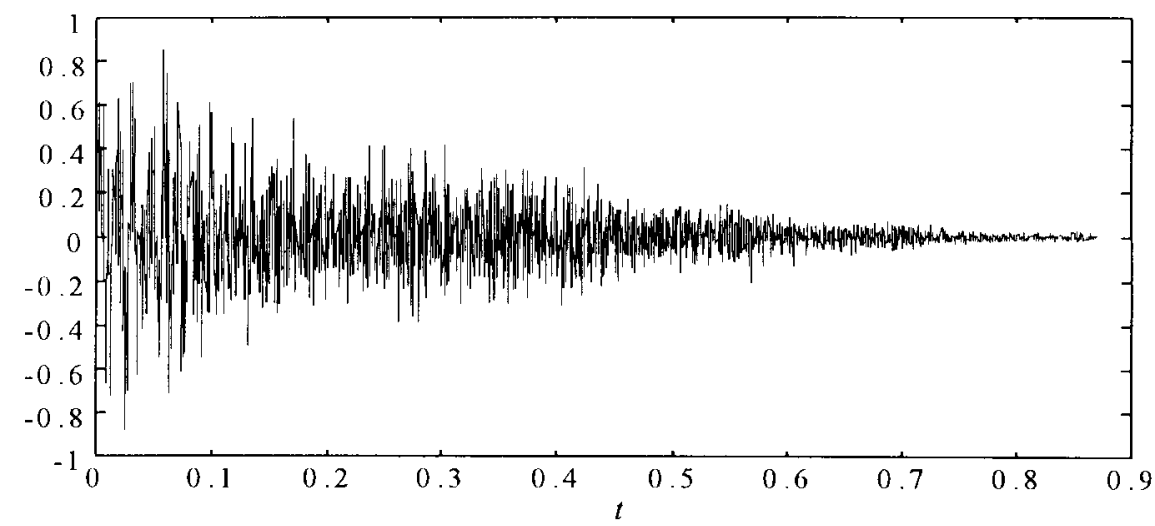

(b)

Fig. 12. PSFWWT analysis of a 27.3-Hz piano tone. (a) Regular component. (b) Hammer noise.

of the inverse of the optimum map, showing a strikingly good agreement with data. We also compared the Laguerre warping curve with the set of eigenfrequencies derived from the physical model of stiff strings, based on fourth-order PDE [4], [5]. We concluded that for a wide range of the physical parameters, there is always a member of the family of Laguerre warping curves that closely approximates the dispersion characteristics of the stiff string.

The pitch parameter $P$ in the PSFWWT must be assigned a value equal to the rounded average period, in number of samples, of the warped signal. Equivalently, one can detect the pitch of the input signal and prewarp this value in order to obtain $P$. The results of the PSFWWT analysis of a piano tone is reported in Fig. 12. There, the regular (scaling) and noisy (wavelet) components are separately inverse transformed, obtaining two distinct signals whose sum is exactly equal to the original sound. In the noisy components, the sound produced by the hammer on the string is highly enhanced. This is vice versa when the regular component contains a "cleanedup" version of the string sound. Clearly, it is possible to separate sounds in multiple wavelet and scaling components, representing different features of the instrument or perceptual characteristics.

By using frequency warped wavelets in place of ordinary wavelets in (35), it is possible to completely control the width of the analysis sidebands of the partials, thus enhancing resolution in the separation of components. Furthermore, it is easy to extend this concept from multiplexed wavelets to multiwavelets [26] in order to obtain separation of the sidebands of each of the partials at the expense of reduced time resolution.

\section{CONCLUSIONS}

In this paper, we presented extensions of the wavelet transform obtained by merging the filterbank structure with the Laguerre transform block. The discrete Laguerre transform provides the unique type of orthogonal warping operator that can be computed using rational transfer functions. We introduced the discrete-time warped wavelets and then showed that continuous-time warped wavelet bases can be generated starting form their discrete-time counterpart. Furthermore, we extended the theory to include pitch-synchronous warped wavelets. Frequency warped wavelet bases have an arbitrary band structure that can be adapted to signals or perceptual characteristics. The flexible time-frequency tiling property of the representation becomes useful in a large class of problems in signal processing, such as signal identification, denoising, and feature detection.

\section{APPENDIX}

In this Appendix, we briefly review the principal properties of the Laguerre sequences [7]-[9], [14], [15] given in the time 


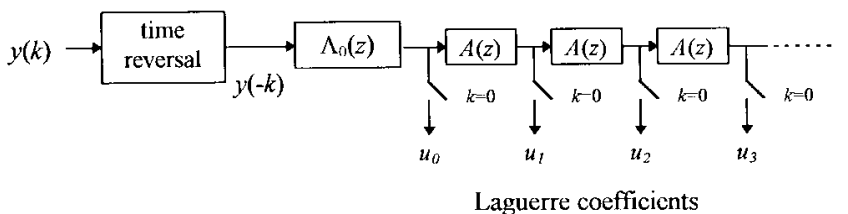

Fig. 13. Structure implementing the Laguerre transform $u_{r}$ of a signal $y(k)$.

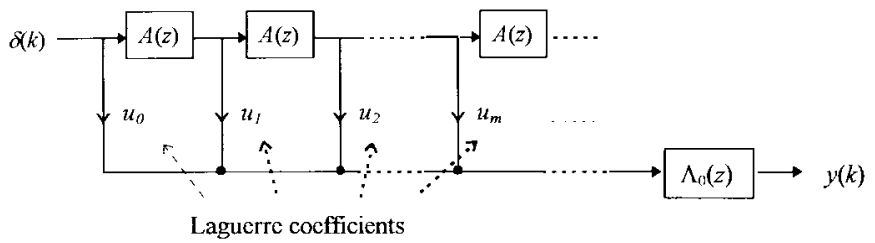

Fig. 14. Structure implementing the inverse Laguerre transform.

domain by the sum

$$
\begin{aligned}
\lambda_{r}(k)= & \sqrt{1-b^{2}} \sum_{m=0}^{\min (r, k)}(-1)^{m+r} \frac{(k+r-m) !}{m !(k-m) !(r-m) !} \\
& \cdot b^{r+k-2 m}, \quad r, k=0,1, \cdots .
\end{aligned}
$$

Laguerre sequences form a complete orthonormal set in $l^{2}(N)$ - extendible to $l^{2}(Z)$ - for any value of the parameter $-1<b<1$. From the $z$ transform of the order- $r$ sequence given by

$$
\Lambda_{r}(z)=\sqrt{1-b^{2}} \frac{\left(z^{-1}-b\right)^{r}}{\left(1-b z^{-1}\right)^{r+1}}
$$

one can see that the Laguerre sequences satisfy the recurrence

$$
\Lambda_{r+1}(z)=A(z) \Lambda_{r}(z)=A(z)^{r+1} \Lambda_{0}(z), \quad r=0,1, \cdots
$$

where

$$
A(z)=\frac{z^{-1}-b}{1-b z^{-1}}
$$

is a stable and causal allpass filter. The order 0 Laguerre sequence is lowpass for $b>0$ and highpass for $b<0$. Since the Laguerre sequences are infinite-length, the Laguerre coefficients can be computed in finite time only if the input is time limited. Furthermore, the transform must be necessarily truncated since only a finite number of Laguerre coefficients can be computed in finite time. It is possible to show [22] that in order to obtain a good accuracy of the representation of a length- $D$ signal, the following lower bound on the number $M$ of Laguerre coefficients must be satisfied.

$$
M>\frac{D(1+|b|)}{1-|b|}
$$

where the first $M$ terms in the Laguerre expansion are retained. In this case, the maximum error is bounded as

$$
e_{\max } \leq B \cdot \max _{n=0, \cdots, D-1}\left\{\sum_{r=0}^{D-1}\left|\delta_{r, n}-\sum_{k=0}^{M-1} \lambda_{k}(r) \lambda_{k}(n)\right|\right\}
$$

where $B$ is the absolute maximum of the signal. The maximum error may be evaluated numerically. When $M$ strictly satisfies
(43), we found $e_{\max }<0.05 \cdot B$. By slightly increasing $M$, the error significantly decreases; for example, if $M$ is $3 \%$ larger, one obtains $e_{\max }<5 \cdot 10^{-4} \cdot B$.

The Laguerre transform may be computed by means of the cascaded allpass structure [10], [11], [14], [15] shown in Fig. 13. Given a finite duration $D$ signal, the expansion coefficients are obtained at the output of each section at the time lag $D$. The inverse Laguerre transform may be computed by means of the Laguerre filter structure shown in Fig. 14. Alternately, one may show that the same structure adopted for computing the Laguerre transform may be used for computing its inverse, provided the sign of the parameter is reversed.

\section{REFERENCES}

[1] K. Hoffman, Banach Spaces of Analytic Functions. New York: Dover, p. 64.

[2] A. Galembo and A. Askenfelt, "Novel methods for the analysis of inharmonicity," Speech Transmission Lab. Quarterly Progress Status Rep., R. Inst. Technol., Stockholm, Sweden, pp. 135-144, 1994.

[3] M. Podlesak and A. R. Lee, "Dispersion of waves in piano strings," $J$. Acoust. Soc. Amer., vol. 83, no. 1, pp. 305-317, Jan. 1988.

[4] P. Morse and K. Ingard, Theoretical Acoustics. New York: McGrawHill, 1968.

[5] J. O. Smith, III, "Physical modeling using digital waveguides," Comput. Music J., vol. 16, no. 4, pp. 74-87, 1992.

[6] G. Evangelista and S. Cavaliere, "The discrete-time frequency warped wavelet transforms," in Proc. ICASSP, Munich, Germany, Apr. 1997.

[7] T. Oliveira e Silva, "On the determination of the optimal pole position of Laguerre filters," IEEE Trans. Signal Processing, vol. 43, pp. 2079-2087, Sept. 1995.

[8] _ "Optimality conditions for truncated Laguerre networks," IEEE Trans. Signal Processing, vol. 42, pp. 2528-2530, Sept. 1994.

[9] C. Braccini and A. V. Oppenheim, "Unequal bandwidth spectral analysis using digital frequency warping," IEEE Trans. Acoust., Speech Signal Processing, vol. ASSP-22, pp. 236-244, Aug. 1974.

[10] A. V. Oppenheim and D. H. Johnson, "Discrete representation of signals," Proc. IEEE, vol. 60, pp. 681-691, June 1972.

[11] A. V. Oppenheim, D. H. Johnson, and K. Steiglitz, "Computation of spectra with unequal resolution using the fast Fourier transform," Proc. IEEE, vol. 59, pp. 299-301, Feb. 1971.

[12] R. G. Baraniuk and D. L. Jones, "Warped wavelets bases: Unitary equivalence and signal processing," in Proc. IEEE Int. Conf. Acoust., Speech, Signal Process, ICASSP, 1993, vol. III, pp. 320-323.

[13] R. G. Baraniuk and D. L. Jones, "Unitary equivalence: A new twist on signal processing," IEEE Trans. Signal Processing, vol. 43, pp. 2269-2282, Oct. 1995.

[14] P. W. Broome, "Discrete orthonormal sequences," J. Assoc. Comput. Mach., vol. 12, no. 2, pp. 151-168, 1965.

[15] M. A. Masnadi-Shirazi, "Optimum Laguerre networks for a class of discrete-time systems," IEEE Trans. Signal Processing, vol. 39, pp. 2104-2108, Sept. 1991.

[16] P. P. Vaidyanathan, "Multirate digital filters, filter banks, polyphase networks, and applications: A tutorial," Proc. IEEE, vol. 78, pp. 56-93, 1990.

[17] M. Vetterli, "A theory of multirate filter banks," IEEE Trans. Acoust., Speech, Signal Processing, vol. ASSP-35, pp. 356-372, 1987.

[18] I. Daubechies, "Orthonormal bases of compactly supported wavelets," Commun. Pure Appl. Math., vol. XLI, no. 7, pp. 909-996, 1988.

[19] G. Evangelista, "Comb and multiplexed wavelet transforms and their applications to signal processing," IEEE Trans. Signal Processing, vol. 42, pp. 292-303, Feb. 1994.

[20] _ "Pitch synchronous wavelet representations of speech and music signals," IEEE Trans. Signal Processing, vol. 41, pp. 3313-3330, Dec. 1993.

[21] _ "The coding gain of multiplexed wavelet transforms," IEEE Trans. Signal Processing, vol. 44, pp. 1681-1692, July 1996.

[22] G. Evangelista and S. Cavaliere, "Frequency warped filter banks and wavelet transforms: A discrete-time approach via Laguerre expansion," submitted for publication.

[23] T. Blu, "Iterated filter banks with rational rate changes connection with discrete wavelet transform," IEEE Trans. Signal Processing, vol. 41, pp. 3232-3244, Dec. 1993. 
[24] C. Herley, J. Kovacevic, K. Ramchandran, and M. Vetterli, "Tilings of the time-frequency plane: Construction of arbitrary orthogonal bases and fast tiling algorithms," IEEE Trans. Signal Processing, vol. 41, pp. 3341-3359, Dec. 1993

[25] R. R. Coifman and M. V. Wickerhauser, "Entropy-based algorithms for best basis selection," IEEE Trans. Inform. Theory, vol. 38, pp. 713-718, Mar. 1992

[26] G. Strang and V. Strela, "Orthogonal multiwavelets with vanishing moments," J. Opt. Eng., vol. 33, pp. 2104-2107, 1994

[27] G. W. Wornell and A. V. Oppenheim, "Wavelet-based representations for a class of self-similar signals with applications to fractal modulation," IEEE Trans. Inform. Theory, vol. 38, pp. 785-800, Mar. 1992.

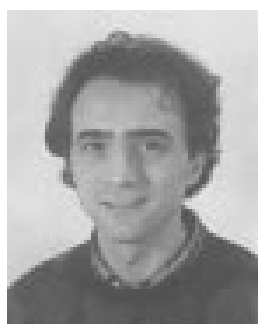

Gianpaolo Evangelista (M'92) received the laurea in physics (summa cum laude) from the University of Napoli, Napoli, Italy, in 1984 and the M.Sc. and $\mathrm{Ph} . \mathrm{D}$. degrees in electrical engineering from the University of California, Irvine, in 1987 and 1990, respectively.

$\mathrm{He}$ is a Researcher at the Department of Physical Sciences, University of Napoli Federico II, Napoli. From 1985 to 1986 , he worked at the Centre d'Etudes de Mathématique et Acoustique Musicale (CEMAMu/CNET), Paris, France, where he contributed to the development of a DSP-based sound synthesis system, and from 1991 to 1994, he was a Research Engineer at the Microgravity Advanced Research and Support (MARS) Center, Napoli, where he was engaged in research in image processing applied to fluid motion analysis. His interests include speech, music, and image processing; coding; wavelets; and multirate signal processing.

Dr. Evangelista was a recipient of the Fulbright fellowship.

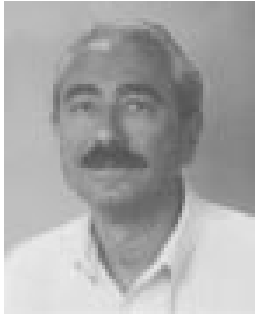

Sergio Cavaliere received the laurea in electronic engineering (summa cum laude) from the University of Napoli Federico II, Napoli, Italy, in 1971.

From 1972 to 1973 , he was with CNR at the University of Siena, and since 1974, he has been a Research Associate with the Department of Physical Sciences, University of Napoli. In 1986, he spent an academic year at the Media Laboratory, Massachusetts Institute of Technology, Cambridge. From 1987 to 1991 , he received a research grant for a project devoted to the design of VLSI chips for real-time sound processing and for the realization of the Musical Audio Research Station (MARS) workstation for sound manipulation, IRIS, Rome, Italy. Currently, he is also a Research Associate with INFN for the realization of very-large systems for data acquisition from nuclear physics experiments and for the development of techniques for the detection of signals in high level noise. His interests include sound and music signal processing, signal transforms and representations, VLSI, and specialized computers for sound manipulation. 